\section{Revista de CIENCIAS AMBIENTALES Tropical Journal of Environmental Sciences}

Revista de Ciencias Ambientales (Trop J Environ Sci)

e-ISSN: 2215-3896

(Julio-Diciembre, 2019) . Vol 53(2): 97-110

DOI: https://doi.org/10.15359/rca.53-2.5

Open Access: www.revistas.una.ac.cr/ambientales e-mail: revista.ambientales@una.ac.cr Centeno E. y Murillo A.

\title{
Tipología de las tecnologías de tratamiento de aguas residuales ordinarias instaladas en Costa Rica
}

\author{
Typology of Ordinary Wastewater Treatment Technologies Installed in Costa Rica
}

\author{
Erick Centeno Mora ${ }^{a}$ y Adrián Murillo Marín ${ }^{b}$
}

[Recibido: 13 de enero 2019, Aceptado: 9 de abril 2019, Corregido: 17 de abril 2019, Publicado: 1 de julio 2019]

\section{Resumen}

Este artículo presenta el perfil de las plantas de tratamiento de aguas residuales ordinarias en Costa Rica, comparándolo con el de varios países de América Latina y el Caribe. Con datos de 47 plantas administradas por entidades públicas, complementados con el análisis de una muestra de 382 sistemas tramitados para su construcción entre el 2011 y el 2016, se caracterizó la tipología de las plantas de tratamiento de aguas residuales promovidas en territorio costarricense, en términos de capacidad instalada y tecnología utilizada. Los resultados muestran que más del $90 \%$ de los sistemas operados por entidades públicas puede ser considerado de pequeña capacidad, con 70\% concebido para caudales inferiores a $5.0 \mathrm{~L} / \mathrm{s}$. En el caso de los proyectos tramitados para su construcción, más del $90 \%$ de las plantas tiene una capacidad menor a $5.0 \mathrm{~L} / \mathrm{s}$ y $84 \%$ corresponde a sistemas para condominios privados. Con respecto a las tecnologías más usadas en el país, el número de plantas que emplean lodos activados representa la mayoría, con $57 \%$ de los sistemas operado por entidades públicas y $98 \%$ de los proyectos tramitados para su construcción. Costa Rica tiene una proporción de sistemas de pequeña capacidad ( $\mathrm{Q}<25 \mathrm{~L} / \mathrm{s})$ y utiliza la tecnología de lodos activados en mayor medida que los otros países de la región contemplados en este análisis. Estas 2 condiciones pueden resultar en costos de operación más elevados, por lo que se recomienda evaluar dicha práctica, con el fin de promover un saneamiento universal y más sostenible.

Palabras clave: estaciones depuradoras; infraestructura de saneamiento; planta de tratamiento; sostenibilidad; tecnologías apropiadas.

\begin{abstract}
This paper presents a profile of municipal wastewater treatment plants in Costa Rica, comparing it with those of other Latin American and Caribbean countries. By collecting data from 47 plants operated by public entities, complemented by the analysis of a sample of 382 projects which had requested construction permits between 2011 and 2016, it was possible to characterize wastewater treatment in Costa Rica in terms of capacity and technology trends. Results show that more than $90 \%$ of the plants operated by public entities can be considered as small, with $70 \%$ designed for flows of less than $5.0 \mathrm{~L} / \mathrm{s}$. With regard to the projects which had requested construction permits, more than $90 \%$ of the plants were designed for flows of less than $5.0 \mathrm{~L} / \mathrm{s}$, and $84 \%$ were systems for private condominium projects. Use of activated sludge in treatment technology was found in $57 \%$ of the systems operated by public entities and in $98 \%$ the projects which had requested construction permits. Costa Rica has a higher proportion of small capacity plants $(\mathrm{Q}<25 \mathrm{~L} / \mathrm{s})$ and uses activated sludge more frequently than the other Latin American and Caribbean countries that were analyzed in this study. These two conditions can result in higher operating costs, and it is recommended to review this practice in order to promote universal and more sustainable sanitation.
\end{abstract}

Keywords: appropriate technologies; sanitation infrastructure; sewage treatment plants; sustainability.

a Profesor Escuela de Ingeniería Civil, Universidad de Costa Rica, Costa Rica, erick.centenomora@ucr.ac.cr, https://orcid. org/0000-0001-8806-0355

b Ingeniero de proyecto, Ingeniería y Administración S.A., amurillo@iaacr.com, https://orcid.org/0000-0002-2927-3569

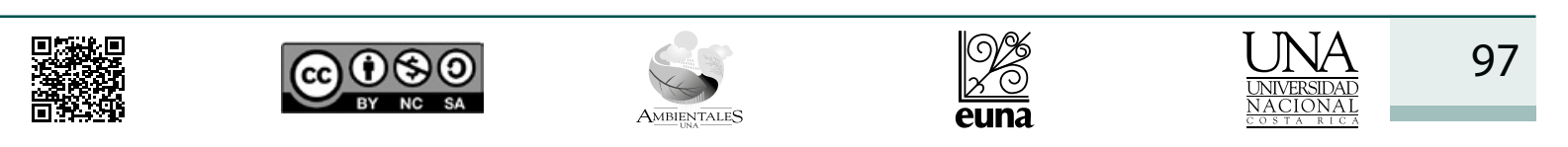




\section{Revista de CIENCIAS AMBIENTALES Tropical Journal of Environmental Sciences}

Revista de Ciencias Ambientales (Trop J Environ Sci) e-ISSN: 2215-3896

(Julio-Diciembre, 2019) . Vol 53(2): 97-110 DOI: https://doi.org/10.15359/rca.53-2.5

Open Access: www.revistas.una.ac.cr/ambientales e-mail: revista.ambientales@una.ac.cr Centeno E. y Murillo A.

\section{Introducción}

Los Objetivos del Desarrollo Sostenible (ODS) de la Organización de las Naciones Unidas, específicamente en el 6 y de manera indirecta en $\operatorname{los} 3,7,11$ y 15, promueven la universalización del saneamiento en aguas residuales de modo sostenible, reduciendo la proporción de aguas residuales no tratadas y promoviendo tanto el reúso como la valorización de subproductos del saneamiento (UN WATER, 2017).

En concordancia con los ODS, Costa Rica, en su Política Nacional de Saneamiento en Aguas Residuales 2016-2045 (Instituto Costarricense de Acueductos y Alcantarillados [AyA], Ministerio de Ambiente y Energía [MINAE] y Ministerio de Salud [MINSA], 2016), establece una serie de objetivos y metas entre los que destaca la recolección y el tratamiento del $100 \%$ de las aguas residuales en las áreas de alta densidad poblacional para el 2045. Dado que en el 2017 únicamente un $28.5 \%$ de la población urbana contaba con alcantarillado sanitario y solo $8.2 \%$ de las aguas residuales colectadas recibía algún tipo de tratamiento (Herrera, 2017), el reto que se le plantea al país es considerable. Según estimaciones del AyA et al., (2016), el caudal de aguas residuales adicional que debería ser colectado y tratado al 2045, solo en las zonas urbanas del país, ascendería a aproximadamente $6.2 \mathrm{~m}^{3} / \mathrm{s}$, sin considerar el aumento en la población futura.

En investigaciones previas, se ha estudiado el tipo de saneamiento existente en Costa Rica (individual o colectivo) y su evolución, así como la cobertura en alcantarillado sanitario público (Angulo, 2014; Arias, 2010; Astorga y Angulo, 2013; Herrera, 2016). En otras indagaciones, se han caracterizado los proyectos de plantas de tratamiento de aguas residuales (PTAR) que se tramitan en el nivel de permisos de construcción (Campos, 2015), los cuales informan sobre la capacidad y las tecnologías de uso más común en el país. Sin embargo, aún se carece de un perfil del tratamiento de las aguas residuales, en términos de capacidad de los sistemas y tecnologías instaladas que permitan comparar la situación nacional con otras naciones de la región latinoamericana.

El objetivo de este artículo es establecer la tipología de las PTAR ordinarias existentes en Costa Rica, en términos de capacidad de los sistemas y de las tecnologías instaladas, identificando patrones en el tratamiento de las aguas residuales. Al comparar la condición costarricense con la de otros países de la región latinoamericana, se espera contribuir a una mejor comprensión de la realidad nacional en el sector del saneamiento, que contribuya a señalar retos y oportunidades en la ruta hacia un saneamiento universal y sostenible.

\section{Metodología}

El análisis de la tipología de las PTAR en Costa Rica se realizó a partir de 2 bases de datos distintas. La primera de ellas consistió en una compuesta por los sistemas de tratamiento de aguas residuales ordinarias operados por entidades públicas. La información se recolectó a partir de entrevistas personales y telefónicas con los encargados de la administración de las PTAR. Se recopiló información del AyA, la Empresa de Servicios Públicos de Heredia (ESPH), Asociaciones Administradoras de los Sistemas de Acueductos y Alcantarillados comunales (ASADAS) y municipalidades. La muestra de sistemas incluidos en esta base de datos fue de 47 PTAR de

\begin{tabular}{|c|c|c|c|c|c|}
\hline 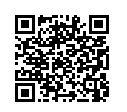 & (c) (i) (-) & $\overbrace{\text { AMBENETIES }}$ & $\frac{9 \%}{\text { euna }}$ & 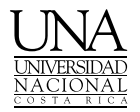 & 98 \\
\hline
\end{tabular}




\section{Revista de CIENCIAS AMBIENTALES Tropical Journal of Environmental Sciences}

Revista de Ciencias Ambientales (Trop J Environ Sci) e-ISSN: 2215-3896

(Julio-Diciembre, 2019) . Vol 53(2): 97-110 DOI: https://doi.org/10.15359/rca.53-2.5

Open Access: www.revistas.una.ac.cr/ambientales e-mail: revista.ambientales@una.ac.cr Centeno E. y Murillo A.

un total en todo el país estimado en 58 (Mora, Mata y Portuguez, 2016), que resulta en un $81 \%$ de la población. El detalle de la composición de dicha base de datos se muestra en el Cuadro 1, incorporando su distribución espacial por provincia.

Cuadro 1. PTAR administradas por entidades públicas en Costa Rica, por provincia, según entidad pública administradora

\begin{tabular}{llllll}
\hline Provincia & \multicolumn{2}{l}{ Ente administrador } & & \\
\hline & AyA & Municipalidades & ASADAS & ESPH & Total \\
Alajuela & 2 & 11 & 2 & - & 15 \\
Cartago & - & - & 1 & - & 1 \\
Guanacaste & 4 & - & - & - & 4 \\
Heredia & 2 & 3 & 1 & 5 & 11 \\
Limón & 2 & - & 2 & - & 4 \\
Puntarenas & 3 & - & - & - & 3 \\
San José & 8 & - & 1 & - & 9 \\
\hline PTAR analizadas en artículo & $\mathbf{2 1}$ & $\mathbf{1 4}$ & $\mathbf{7}$ & $\mathbf{5}$ & $\mathbf{4 7}$ \\
\hline PTAR en operación en & $\mathbf{2 1}$ & $\mathbf{2 2}$ & $\mathbf{1 0}$ & $\mathbf{5}$ & $\mathbf{5 8}$ \\
el país según Mora $e$ al., (2016) & & & & & \\
\hline
\end{tabular}

Fuente: Adaptado de Murillo (2017).

La segunda base de datos analizada correspondió a una parte de los proyectos de PTAR de aguas ordinarias tramitados en la herramienta del Colegio Federado de Ingenieros y Arquitectos (CFIA) de Costa Rica, conocida como Administrador de Proyectos de Construcción (APC). Todos los nuevos proyectos de construcción de PTAR deben ser inscritos en el APC desde el 2011. La base estudiada contaba con información desde el 2011 hasta agosto del 2016, datos de 382 PTAR de un total de 1830 sistemas tramitados entre el 2007 y el 2015 (Herrera, 2016), para un porcentaje de $21 \%$ del total de PTAR de aguas residuales ordinarias manejadas en Costa Rica. No existe garantía de que todos los proyectos gestionados en el APC se construyeron y están actualmente en operación; sin embargo, la herramienta permite obtener información sobre las tendencias de los desarrolladores (principalmente privados) con respecto al tamaño y la tecnología de los sistemas de tratamiento de aguas residuales ordinarias que se ejecutan y se construyen en el país.

Para ambas bases de datos, las PTAR fueron clasificadas según su capacidad (en términos de caudal o por medio de la población servida) y su tecnología de tratamiento, agrupándolas en función de las tecnologías institucionalizadas en Costa Rica: lodos activados con aireación extendida (LAAE), sistemas lagunares, sistemas anaerobios (agrupando reactores anaerobios de flujo ascendente de manto de lodo, conocidos como reactores UASB, y filtros anaerobios de flujo ascendente, denominados FAFA), emisario submarino, tratamiento primario, sistema integrado

\begin{tabular}{|c|c|c|c|c|}
\hline 唃要 & (c) (i) (-) () & 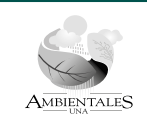 & $\frac{O \%}{2 \%}$ & 99 \\
\hline
\end{tabular}




\section{Revista de CIENCIAS AMBIENTALES Tropical Journal of Environmental Sciences}

Revista de Ciencias Ambientales (Trop J Environ Sci) e-ISSN: $2215-3896$

(Julio-Diciembre, 2019) . Vol 53(2): 97-110 DOI: https://doi.org/10.15359/rca.53-2.5 Open Access: www.revistas.una.ac.cr/ambientales e-mail: revista.ambientales@una.ac.cr Centeno E. y Murillo A.

de lodos activados (IFAS), filtro percolador y humedales artificiales. Se excluyó del análisis los tanques sépticos $\mathrm{u}$ otras soluciones individuales de tratamiento o disposición de las excretas. Del mismo modo, del estudio se excluyeron los sistemas de tratamiento de aguas especiales. Para convertir la capacidad de las PTAR de caudal a población equivalente, se consideró una producción de aguas residuales per cápita de $200 \mathrm{~L} /$ persona-d (AyA et al., 2016).

En este análisis, no se consideró la calidad del efluente o el cumplimiento de los sistemas de tratamiento existentes con los límites de vertido establecidos en el Reglamento de Vertido y Reuso de Aguas Residuales (DE-33601-s-MINAE), ni se recuperaron costos de construcción u operación de los proyectos.

\section{Resultados}

En primer lugar, se presentan los resultados para los sistemas operados por entidades públicas, para, posteriormente, exponer los derivados de la base de datos del APC que considera públicos y privados.

\subsection{PTAR operadas por entidades públicas}

En el Cuadro 2 se observa el número de PTAR operadas por entidades públicas según su capacidad. Se consideró la clasificación usada por Noyola et al., (2012) para caracterizar las PTAR de diversos países de América Latina, propuesta por el Centro Panamericano de Ingeniería Sanitaria y Ciencias del Ambiente (CEPIS).

Cuadro 2. Clasificación de las PTAR operadas por entes públicos según capacidad

\begin{tabular}{llll}
\hline $\begin{array}{l}\text { Clasificación según el } \\
\text { tamaño de la PTAR }\end{array}$ & Rango de caudal $(\mathrm{L} / \mathbf{s})$ & Cantidad de sistemas & Porcentaje \\
\hline Pequeñas & De 0 a 25.0 & 43 & 91.5 \\
\hline & De 0 a 5.0 & 32 & \\
\multicolumn{1}{c}{ Detalle de sistemas } & De 5.1 a 10.0 & 3 & \\
pequeños & De 10.1 a 15.0 & 4 & \\
& De 15.1 a 20.0 & 2 & \\
\hline Medianas & De 20.1 a 25.0 & 2 & 6.4 \\
Grandes & De 25.1 a 250 & 3 & 0 \\
Muy grandes & De 250 a 2500 & 0 & 2.1 \\
\hline Total & $>2500$ & 1 & $\mathbf{1 0 0}$ \\
\hline
\end{tabular}

Fuente: Adaptado de Murillo (2017).

El Cuadro 2 muestra que más del $91 \%$ de los sistemas operados por entidades públicas entra en el rango de pequeño, un $6 \%$ de los sistemas es mediano y apenas un sistema entraría

(cc)




\section{Revista de CIENCIAS AMBIENTALES Tropical Journal of Environmental Sciences}

Revista de Ciencias Ambientales (Trop J Environ Sci) e-ISSN: 2215-3896

(Julio-Diciembre, 2019) . Vol 53(2): 97-110 DOI: https://doi.org/10.15359/rca.53-2.5

Open Access: www.revistas.una.ac.cr/ambientales e-mail: revista.ambientales@una.ac.cr Centeno E. y Murillo A.

en la clasificación de muy grande (la PTAR de los Tajos, operada por el AyA). Existe un salto de capacidad entre esta PTAR (2 $810 \mathrm{~L} / \mathrm{s})$ y el resto de sistemas operados en el país; la PTAR del Roble de Puntarenas, también operada por el AyA, es la que le sigue en capacidad ( $90 \mathrm{~L} / \mathrm{s})$. A la fecha, no hay en Costa Rica PTAR que entren en la clasificación de sistemas grandes (de 250 a 2 $500 \mathrm{~L} / \mathrm{s}$ ). Entre los sistemas pequeños, más del $70 \%$ tiene una capacidad inferior a $5.0 \mathrm{~L} / \mathrm{s}$, lo que equivale a la producción de aguas residuales de aproximadamente 2160 habitantes.

En la Figura 1, se muestra la capacidad instalada y la cantidad de PTAR por tecnología de tratamiento para los sistemas de tratamiento administrados por entidades públicas. Entre los sistemas anaerobios, se agruparon reactores UASB con y sin postratamiento, así como sistemas de Sedimentador Digestor con FAFA. Del mismo modo, los sistemas lagunares agrupan lagunas facultativas y otras con algún grado de aireación mecánica. No se contaba con información de la tecnología para 3 de los sistemas analizados, por lo que en la Figura 1 se incluyó 43 de las 47 PTAR administradas por entidades públicas.

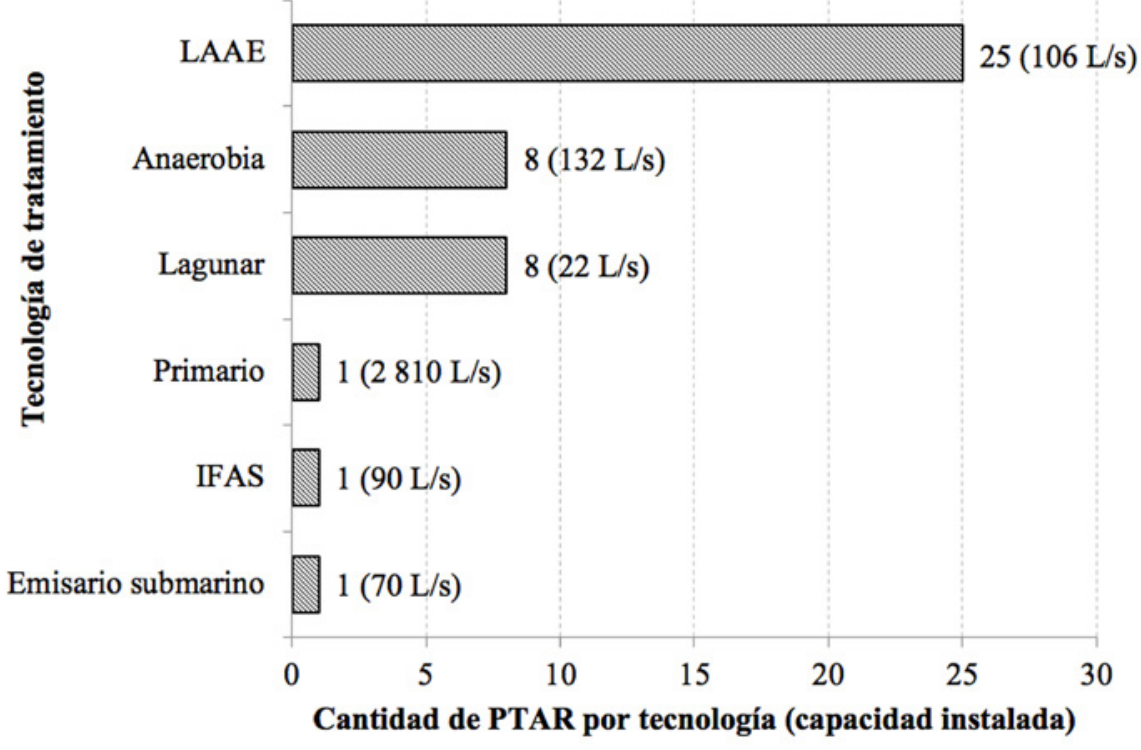

Figura 1. Distribución de tecnologías de tratamiento de aguas residuales según cantidad de PTAR y capacidad instalada (entre paréntesis). Fuente: Adaptado de Murillo (2017).

Como se mencionó, una sola PTAR tiene la mayor capacidad de tratamiento. Se trata de Los Tajos, con tratamiento primario. La segunda tecnología con mayor capacidad instalada en el nivel de entes operadores públicos son los sistemas lagunares $(132 \mathrm{~L} / \mathrm{s})$, que ocupan el tercer lugar en cuanto al número de sistemas instalados (8 plantas). Destaca el hecho de que la tecnología de lodos activados de aireación extendida (LAAE) es, por un amplio margen, la que posee mayor número de sistemas instalados (25 plantas, para un $57 \%$ de las PTAR en la muestra) que

\begin{tabular}{|c|c|c|}
\hline 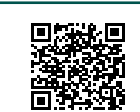 & (c) & 101 \\
\hline
\end{tabular}




\section{Revista de CIENCIAS AMBIENTALES Tropical Journal of Environmental Sciences}

Revista de Ciencias Ambientales (Trop J Environ Sci) e-ISSN: 2215-3896

(Julio-Diciembre, 2019) . Vol 53(2): 97-110 DOI: https://doi.org/10.15359/rca.53-2.5

Open Access: www.revistas.una.ac.cr/ambientales e-mail: revista.ambientales@una.ac.cr Centeno E. y Murillo A.

le permiten alcanzar el tercer puesto en el nivel de caudal tratado $(106 \mathrm{~L} / \mathrm{s})$. El Sistema Integrado de Lodos Activados (IFAS) del Roble de Puntarenas, una variante de los sistemas de lodos activados, y el emisario submarino de Limón son 2 tecnologías de elevada capacidad (PTAR medianas), instaladas en un único sistema de tratamiento en Costa Rica. Los sistemas anaerobios ocupan el último lugar en caudal instalado $(22 \mathrm{~L} / \mathrm{s})$, aunque están equiparados en el segundo y tercer lugar, con respecto a la cantidad de sistemas existentes (8 PTAR).

En lo referente a la administración de las PTAR operadas por entidades públicas, el AyA dirige los 6 sistemas de tratamiento de mayor capacidad instalada, seguido por la Municipalidad de Alajuela y la ESPH (cada una con un sistema de capacidad cercana a $20 \mathrm{~L} / \mathrm{s}$ ). Las PTAR de menor capacidad son operadas por ASADAS y municipalidades.

\subsection{PTAR incluidos en la base de datos del APC}

Según la muestra de la base de datos del APC analizado, relacionada con las PTAR inspeccionadas por el AyA en busca de su aprobación, $84 \%$ de las PTAR tramitadas fueron concebidas para condominios (260 sistemas), mientras que el restante 16\% correspondió a sistemas para urbanizaciones y otros desarrollos similares (51 sistemas). Estos resultados concuerdan con los hallados por Campos (2015), cuando analizó la tendencia entre el 2011 y el 2013, lo que muestra que esta proyección se mantuvo durante el período del 2013 al 2016. El modo de disposición final del efluente tratado se muestra en el Cuadro 3, para los proyectos tramitados en el APC, hasta agosto del 2016.

Cuadro 3. Número de proyectos privados de PTAR tramitados en el APC, según tipo de disposición final del efluente tratado, hasta agosto del 2016

\begin{tabular}{lll}
\hline $\begin{array}{l}\text { Tipo de disposición final del } \\
\text { efluente tratado }\end{array}$ & Cantidad & Porcentaje \\
\hline Cuerpo receptor & 234 & 77 \\
Reúso & 55 & 18 \\
Infiltración & 10 & 3 \\
Alcantarillado & 6 & 2 \\
\hline Total & $\mathbf{3 0 5}$ & $\mathbf{1 0 0}$ \\
\hline
\end{tabular}

A partir del Cuadro 3, es posible observar que más de 3 cuartas partes de los proyectos proponen la disposición del efluente en un cuerpo receptor, mientras que 1 quinta parte hizo reúso del agua tratada. La infiltración en el suelo y el alcantarillado sanitario representaron una proporción de apenas $5 \%$ de la muestra. Los requisitos de calidad para el vertido en cuerpo de agua son superiores a los de reúso, infiltración en el suelo o vertido en alcantarillado (DE33601-S-MINAE, 2007). Esta tendencia es congruente con lo que Campos (2015) encontró para el período entre el 2011 y el 2013.

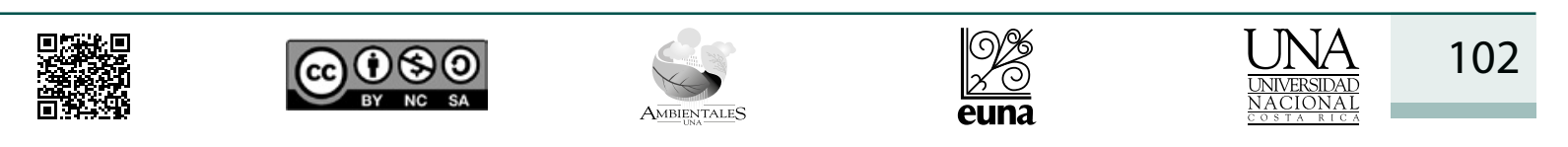




\section{Revista de CIENCIAS AMBIENTALES Tropical Journal of Environmental Sciences}

Revista de Ciencias Ambientales (Trop J Environ Sci) e-ISSN: 2215-3896

(Julio-Diciembre, 2019) . Vol 53(2): 97-110 DOI: https://doi.org/10.15359/rca.53-2.5

Open Access: www.revistas.una.ac.cr/ambientales e-mail: revista.ambientales@una.ac.cr Centeno E. y Murillo A.

En la Figura 2, se observa la distribución en la capacidad de los sistemas de tratamiento de aguas residuales de la muestra de proyectos tramitados en el APC analizado. En el gráfico, no se incluyeron 86 proyectos de la base de datos que no aplicaban por diversos motivos: no tenían el dato de caudal, eran sistemas que no requerían revisión en el APC por parte de AyA, no completaron el trámite, entre otros.

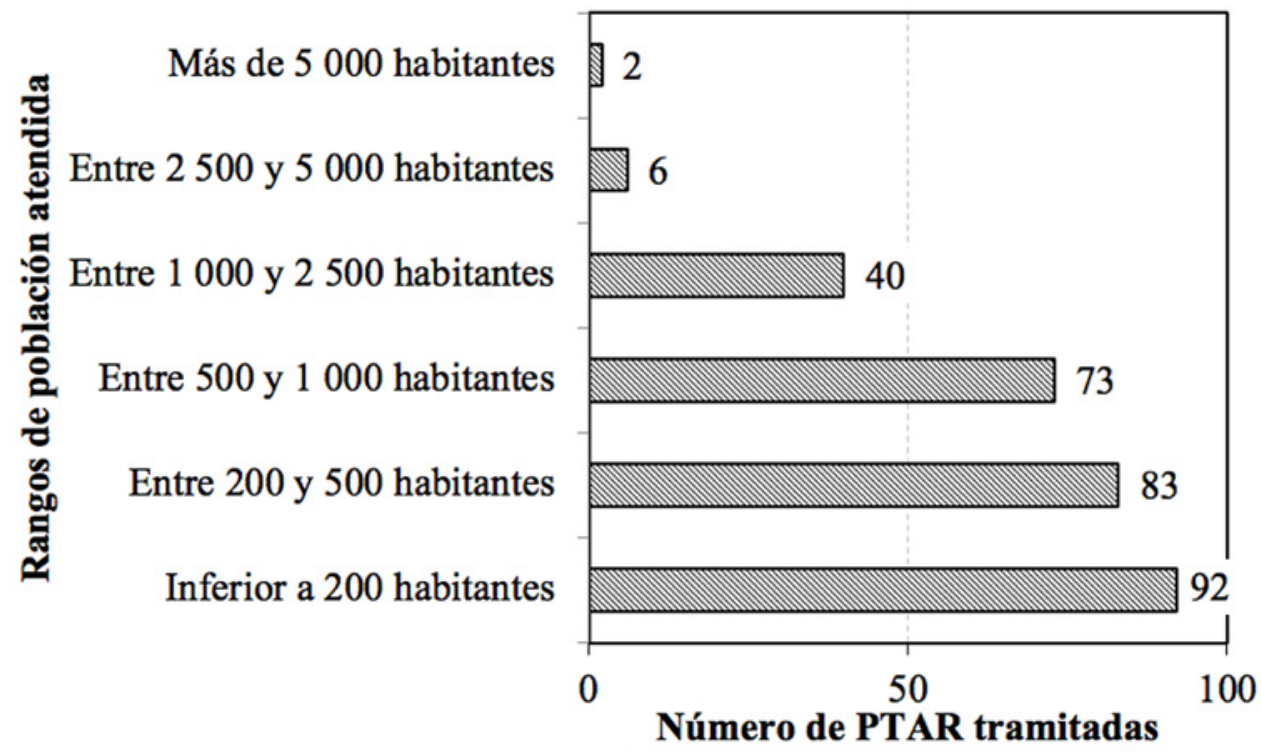

Figura 2. Rangos de población atendida para las plantas de tratamiento de aguas residuales tramitadas en el APC, hasta agosto del 2016.

Se observa que, siguiendo un patrón similar al de los sistemas operados por entidades públicas, al menos 99 \% de los sistemas tramitados en el APC (que consideran tanto proyectos públicos como privados) entra en la categoría de pequeños propuesta por Noyola et al., (2012), con un caudal inferior a $25 \mathrm{~L} / \mathrm{s}$. Adicionalmente, cerca de más de $90 \%$ de los sistemas tiene un caudal inferior a $5.0 \mathrm{~L} / \mathrm{s}$ (población servida de aproximadamente 2160 habitantes equivalentes).

La Figura 3 muestra los resultados obtenidos al agrupar los sistemas, según la tecnología de tratamiento. En esta figura, no se incluyeron 77 proyectos de la base de datos que no aplicaban por razones similares a las expuestas anteriormente.

Es posible observar que más del $98 \%$ de las PTAR de la Figura 3 utiliza la tecnología de lodos activados de aireación extendida (LAAE) o alguna variante de los lodos activados. Apenas $1 \%$ de los proyectos que usaron tecnologías anaerobias y porcentajes menores a la unidad correspondió a sistemas de humedales artificiales o filtros percoladores. Campos (2015) encontró que $97 \%$ de los proyectos tramitados en el APC entre el 2011 y el 2013 consideró alguna variante de la tecnología de lodos activados, lo que muestra que esta tendencia se mantuvo para el período posterior.

\begin{tabular}{|c|c|c|}
\hline 品都票 & (c) (i) (-) & 103 \\
\hline
\end{tabular}




\section{Revista de CIENCIAS AMBIENTALES Tropical Journal of Environmental Sciences}

Revista de Ciencias Ambientales (Trop J Environ Sci) e-ISSN: 2215-3896

(Julio-Diciembre, 2019) . Vol 53(2): 97-110 DOI: https://doi.org/10.15359/rca.53-2.5

Open Access: www.revistas.una.ac.cr/ambientales e-mail: revista.ambientales@una.ac.cr Centeno E. y Murillo A.

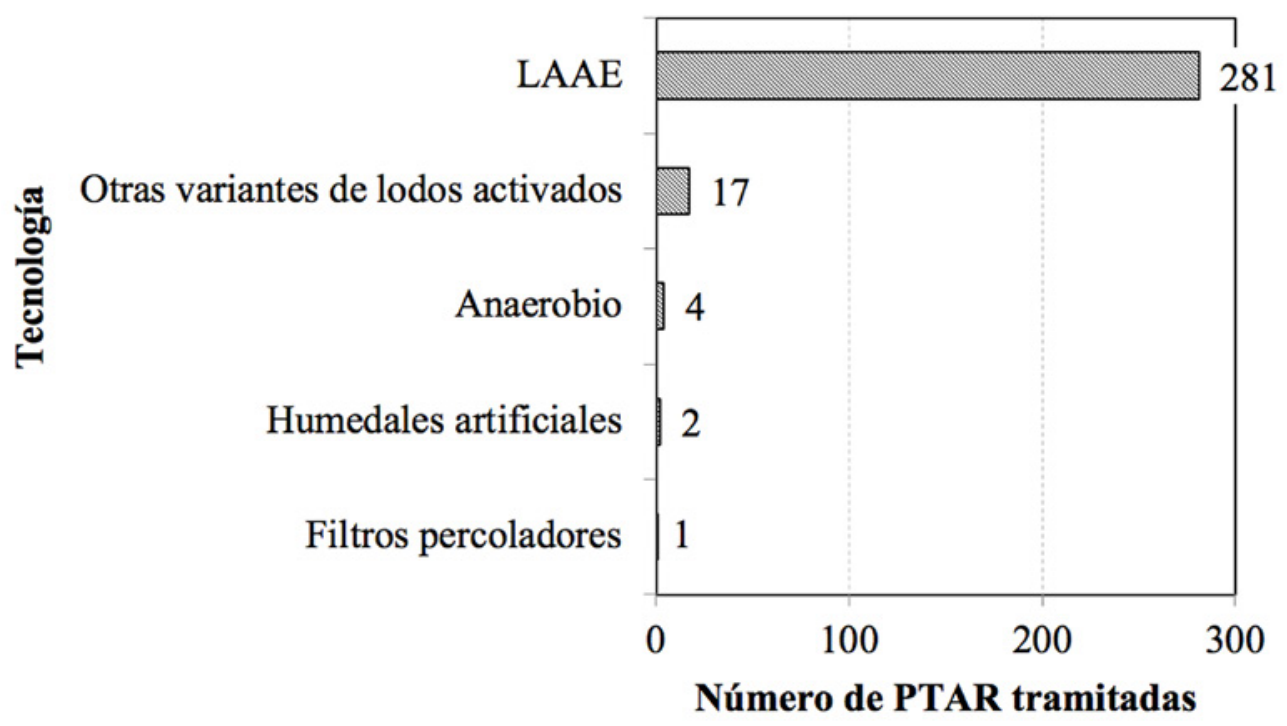

Figura 3. Tecnologías adoptadas para las plantas de tratamiento de aguas residuales tramitadas en el APC, hasta agosto del 2016.

\section{Discusión}

\subsection{Condición del tratamiento de las aguas residuales ordinarias en Costa Rica, en términos de capacidad y tecnología implantada}

A partir de los resultados obtenidos en esta investigación, es posible identificar 2 patrones bien definidos en lo que se refiere a la capacidad de las PTAR y las tecnologías instaladas en Costa Rica. El primero de ellos es que los sistemas de tratamiento tienden a ser de pequeña capacidad, con un alto porcentaje de ellos por debajo de $5.0 \mathrm{~L} / \mathrm{s}$ ( $70 \%$ de los sistemas operados por entidades públicas y $90 \%$ de los sistemas tramitados en el APC que resultaron válidos para este análisis). La importante cantidad de proyectos de PTAR tramitados en el APC con respecto a la cantidad de plantas operadas por entidades públicas, 1830 trámites en el APC, según Herrera (2016), contra 58 PTAR operadas por entidades públicas, de acuerdo con Mora et al. (2016), sugiere que la mayoría de sistemas de tratamiento de aguas residuales ordinarias existentes en el país sería para desarrollos privados.

Esto evidenciaría la adopción de un modelo de saneamiento en aguas residuales descentralizado, con una importante cantidad de sistemas de tratamiento de aguas residuales privados y de pequeña capacidad distribuidos por el país. Según Herrera (2017), la cobertura en alcantarillado sanitario público en el país es de $28.5 \%$ en áreas urbanas, $5.1 \%$ en zonas rurales y todavía un elevado porcentaje de la población (76\%) dispone sus aguas residuales en un sistema

\begin{tabular}{|c|c|c|c|c|c|}
\hline 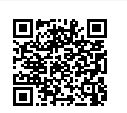 & (c) (i) (-) & $\underset{\text { AMBENTAIES }}{\longrightarrow}$ & $\frac{\mid \sigma o \%}{\text { euna }}$ & 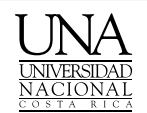 & 104 \\
\hline
\end{tabular}




\section{Revista de CIENCIAS AMBIENTALES Tropical Journal of Environmental Sciences}

Revista de Ciencias Ambientales (Trop J Environ Sci) e-ISSN: 2215-3896

(Julio-Diciembre, 2019) . Vol 53(2): 97-110 DOI: https://doi.org/10.15359/rca.53-2.5

Open Access: www.revistas.una.ac.cr/ambientales e-mail: revista.ambientales@una.ac.cr Centeno E. y Murillo A.

de tanque séptico con infiltración al terreno. De este modo, la baja cobertura en alcantarillado sanitario público y la apuesta histórica a soluciones de saneamiento individual explicarían por qué se cuenta con tantos sistemas de baja capacidad y por qué muchos de ellos son tramitados para desarrollos habitacionales privados, como se comentó.

El otro patrón que es fácilmente identificable es la predominancia de la tecnología de lodos activados en el país, principalmente, su variante de aireación extendida (LAAE), sobre las otras tecnologías. Para los operadores públicos, más del $57 \%$ de los sistemas emplea alguna variante de los lodos activados, mientras que este porcentaje aumenta a $98 \%$, para los proyectos tramitados en el APC. Desarrollados en 1914, en Inglaterra, los lodos activados son la tecnología más usada en el mundo, con miras al tratamiento de las aguas residuales ordinarias (WEF y ASCE, 2017), incluyendo un uso común tanto en sistemas de pequeña capacidad como en plantas de gran tamaño. No existe claridad sobre si la selección de esta tecnología para la mayoría de los sistemas de tratamiento en Costa Rica responde a una evaluación de alternativas tecnológicas dirigida a cada proyecto, o si se debe a una tendencia impulsada por las condiciones de experiencia y mercado locales.

Como numerosas referencias lo confirman (Lema y Suárez-Martínez, 2017; Metcalf y Eddy, 2014; Sperling, 2014; Sperling y Chernicharo, 2002, 2005; WEF y ASCE, 2017), muchas otras tecnologías de tratamiento están disponibles y han sido instaladas exitosamente en países con condiciones climáticas, socioeconómicas y de requisitos de calidad en el efluente similares a las de Costa Rica. Por este motivo, profundizar sobre las causas y la conveniencia de usar casi exclusivamente la tecnología de lodos activados en este territorio debe ser motivo de discusión en el futuro próximo. Normalmente, tanto el nivel socioeconómico de la población servida como la densidad poblacional deben ser aspectos por considerar, a la hora de seleccionar el tipo y la tecnología más adecuados para el tratamiento y la disposición de las aguas residuales (Sperling y Chernicharo, 2005).

\subsection{Comparación del perfil de las PTAR de Costa Rica con el de otros países de América Latina}

Al comparar los resultados obtenidos en esta investigación con los presentados por Noyola et al. (2012), es posible identificar algunas particularidades del tratamiento de aguas residuales en Costa Rica con respecto a otros países de América Latina y el Caribe. Estos autores analizaron una muestra de 1842 PTAR de Brasil, Chile, Colombia, Guatemala, México y República Dominicana.

En cuanto a la capacidad de las PTAR, Noyola et al., (2012) encontraron que, para América Latina, el uso de sistemas de pequeña capacidad (caudal menor a $25 \mathrm{~L} / \mathrm{s}$ ) es muy común, con un $67 \%$ de las plantas de la muestra analizada. Las PTAR con un caudal menor a $5.0 \mathrm{~L} / \mathrm{s}$ representaron el $34 \%$ de los sistemas en América Latina. Los resultados de esta investigación mostraron que el uso de plantas de pequeña capacidad es aún más marcado en Costa Rica, donde más de

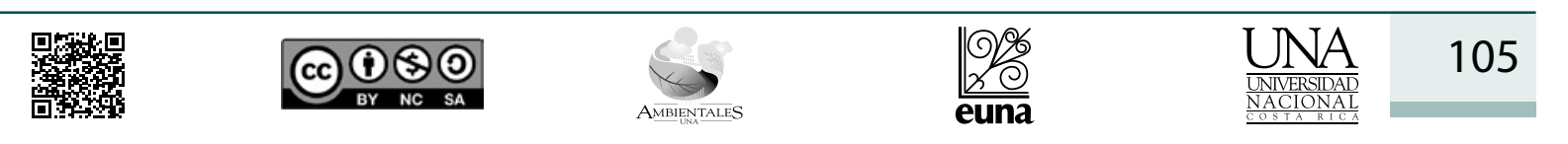




\section{Revista de CIENCIAS AMBIENTALES Tropical Journal of Environmental Sciences}

Revista de Ciencias Ambientales (Trop J Environ Sci) e-ISSN: 2215-3896

(Julio-Diciembre, 2019) . Vol 53(2): 97-110 DOI: https://doi.org/10.15359/rca.53-2.5

Open Access: www.revistas.una.ac.cr/ambientales e-mail: revista.ambientales@una.ac.cr Centeno E. y Murillo A.

$90 \%$ de los sistemas operados por entidades públicas tiene una capacidad inferior a $25 \mathrm{~L} / \mathrm{s}$ y aproximadamente $70 \%$, una inferior a $5.0 \mathrm{~L} / \mathrm{s}$.

Por otro lado, al analizar las tecnologías de tratamiento de las aguas residuales, Noyola et al., (2012) mostraron que existen diferencias marcadas entre los distintos países de América Latina examinados. En general, para la región, las tecnologías más empleadas en función de la cantidad de PTAR son los sistemas lagunares (38 \%), los lodos activados (26\%) y los sistemas anaerobios por medio de reactores UASB (17 \%). En términos de capacidad instalada, los lodos activados ocupan la primera posición ( $58 \%$ ), seguidos de los sistemas lagunares (15\%), los de tratamiento primario mejorado (9\%) y los reactores anaerobios UASB (8 \%). Esta situación difiere significativamente de la condición en Costa Rica, donde los lodos activados son la tecnología más usada en cantidad de sistemas (57\% de los sistemas administrados por entidades públicas y $98 \%$ de los proyectos tramitados en el APC de las muestras analizadas), incluso para PTAR de pequeña capacidad, y en donde la capacidad instalada más importante está dada por un único sistema de tratamiento primario, seguido por los mecanismos lagunares. Por lo tanto, en contra de la tendencia de otros países del área, en la nación costarricense se usa una importante cantidad de sistemas de pequeña capacidad de lodos activados, mientras que en los otros países de América Latina es más común utilizar esa tecnología en las PTAR de mayor capacidad, manteniendo los sistemas lagunares y los anaerobios para las PTAR de menor capacidad.

Chile podría ser el país de la región latinoamericana evaluado por Noyola et al., (2012) con el patrón más similar al de Costa Rica; más del $65 \%$ de los sistemas de tratamiento corresponde a lodos activados y no existen sistemas anaerobios. En el extremo opuesto, se encuentra Brasil, donde menos del $15 \%$ de los sistemas emplea alguna variante de los lodos activados, mientras los sistemas lagunares (40 \%) y los anaerobios UASB (30 \%) son los más usados.

Por último, llama la atención el pobre desarrollo de las tecnologías anaerobias para tratar las aguas residuales ordinarias en Costa Rica, al comparar este país con la región latinoamericana (Noyola et al., 2012). Aunque $21 \%$ de los sistemas operados por entidades públicas aprovechan la tecnología anaerobia (apenas $1 \%$ de los proyectos tramitados en el APC entre el 2011 y el 2016), solo 8.5 \% usa la tecnología UASB; los FAFA son más usuales en el lugar. En otras palabras, la experiencia en el uso de la tecnología UASB es escasa en Costa Rica, aunque en países tropicales como Brasil, Colombia, Guatemala, México y República Dominicana se maneja en el $30 \%, 25 \%, 18 \%, 10 \%$ y $20 \%$ de las PTAR, respectivamente (Noyola et al., 2012). Al complementar esta tecnología con un postratamiento aerobio como los filtros biológicos percoladores (Chernicharo, 2016) o incluso con un sistema de lodos activados, es posible alcanzar efluentes con calidad satisfactoria para su vertido en cuerpos de agua superficiales, a un costo energético y una producción de lodos significativamente menor al de los sistemas de lodos activados como tratamiento biológico único (Chernicharo, van Lier, Noyola y Bressani, 2015).

礔




\section{Revista de CIENCIAS AMBIENTALES Tropical Journal of Environmental Sciences}

Revista de Ciencias Ambientales (Trop J Environ Sci) e-ISSN: 2215-3896

(Julio-Diciembre, 2019) . Vol 53(2): 97-110 DOI: https://doi.org/10.15359/rca.53-2.5

Open Access: www.revistas.una.ac.cr/ambientales e-mail: revista.ambientales@una.ac.cr Centeno E. y Murillo A.

\subsection{Tratamiento de las aguas residuales ordinarias en Costa Rica y costo de operación de los sistemas}

El patrón para el tratamiento de las aguas residuales en Costa Rica, constituido por una gran cantidad de PTAR de pequeña capacidad que emplean mayoritariamente la tecnología de los lodos activados, tiene consecuencias importantes para los costos de operación del sector saneamiento del país.

Rodríguez-García et al., (2011) mostraron que existe un fenómeno de economía de escala, al aumentar la capacidad de los sistemas de tratamiento. Noyola et al., (2012) concluyeron que, a partir de unos $15 \mathrm{~L} / \mathrm{s}$ (aproximadamente 6480 habitantes equivalentes), los sistemas de tratamiento en América Latina mantienen un costo de tratamiento por metro cúbico de agua residual más o menos constante, y este costo unitario es sensiblemente mayor para sistemas de menor capacidad. Esto se debe a la dilución de los precios de personal y a la más factible modulación de los equipos electromecánicos que se consigue en PTAR de mayor capacidad. Consecuentemente, la gran cantidad de sistemas con un caudal de tratamiento inferior a los $5.0 \mathrm{~L} / \mathrm{s}$ en Costa Rica tendría un monto operativo mayor para los usuarios. Adicionalmente, Noyola et al., (2012) sugieren que la operación y el cumplimiento de los límites de vertido serían más difíciles para los sistemas de baja capacidad, por causa de los límites presupuestarios dirigidos al monitoreo de esas PTAR, usualmente más acentuados que para las PTAR de mayor capacidad.

También, el costo de operación de los sistemas de lodos activados, al compararlos con otras tecnologías como los sistemas lagunares o los anaerobios complementados con postratamientos aerobios (incluso con un postratamiento por lodos activados), puede llegar a ser significativamente superior, como reportan Noyola et al., (2012), Sperling y Chernicharo (2005) y Sperling (2014). Esto se debe al consumo energético por parte de los equipos de aireación y a la mayor producción de lodos que requieren un tratamiento y disposición adecuados, en el caso de los lodos activados. De este modo, la predominancia de esta tecnología en Costa Rica también estaría produciendo mayores precios de operación al usuario, que posiblemente no se justifiquen desde un punto de vista técnico, económico o ambiental.

\section{Conclusiones}

El perfil de las PTAR para aguas residuales ordinarias en Costa Rica corresponde a sistemas de pequeña capacidad, donde más de $90 \%$ de los sistemas operados por entidades públicas tiene una capacidad inferior a $25 \mathrm{~L} / \mathrm{s}$ y, aproximadamente, $70 \%$ posee una inferior a $5.0 \mathrm{~L} / \mathrm{s}$. Esta condición se agudiza en el caso de los proyectos tramitados en el APC, pues más del $90 \%$ de las PTAR de la muestra analizada tendría un caudal inferior a $5.0 \mathrm{~L} / \mathrm{s}$. La capacidad limitada de los sistemas de tratamiento estaría vinculada a la baja cobertura en alcantarillado sanitario público en el país, dado que el saneamiento individual, por medio de tanque séptico e infiltración al terreno, es aún mayoritario ( 3 cuartas partes de la población) y la mayoría de iniciativas de proyectos de PTAR corresponden a sistemas para desarrollos privados, principalmente condominios.

\begin{tabular}{|c|c|c|c|c|c|}
\hline 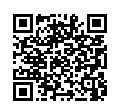 & (c) & $\underset{\text { AMBENTNALES }}{\infty}$ & $\frac{1 \%}{2 \%}$ & $\frac{\text { UNA }}{\frac{\text { UNIVERSIDAD }}{\text { NACIONAL }}}$ & 107 \\
\hline
\end{tabular}




\section{Revista de CIENCIAS AMBIENTALES Tropical Journal of Environmental Sciences}

Revista de Ciencias Ambientales (Trop J Environ Sci) e-ISSN: 2215-3896

(Julio-Diciembre, 2019) . Vol 53(2): 97-110 DOI: https://doi.org/10.15359/rca.53-2.5

Open Access: www.revistas.una.ac.cr/ambientales e-mail: revista.ambientales@una.ac.cr Centeno E. y Murillo A.

Con respecto a las tecnologías más usadas en el país para el tratamiento de las aguas residuales ordinarias, la cantidad mayoritaria de PTAR emplea alguna variante de los lodos activados (57\% de las PTAR operadas por entidades públicas y $98 \%$ de los proyectos tramitados en el APC de las muestras analizadas), aunque la mayor capacidad instalada corresponde a un sistema de tratamiento primario (Los Tajos, aún sin tratamiento biológico), seguido por los sistemas lagunares y los lodos activados. En cuarto lugar de capacidad instalada, se encuentran los sistemas anaerobios, principalmente los FAFA.

Al comparar la tipología del tratamiento de las aguas residuales de Costa Rica con otros países de América Latina, se observa que en la nación costarricense la tendencia es usar sistemas pequeños (capacidad inferior a $25 \mathrm{~L} / \mathrm{s}$ ) y tecnología de lodos activados, la cual es más acentuada que en lugares como Brasil, Chile, Colombia, Guatemala, México y República Dominicana; el uso de la tecnología de reactores anaerobios UASB es menos común.

Tanto la proliferación de múltiples sistemas de pequeña capacidad como el empleo generalizado de la tecnología de lodos activados para tratar las aguas residuales ordinarias son condiciones que generan un mayor costo de operación para personas usuarias, el cual no responde a una planificación en el área del tratamiento de las aguas residuales o a una política nacional de saneamiento.

Un aumento en la cobertura del alcantarillado sanitario con la construcción de sistemas de mayor capacidad, junto con una selección de la tecnología de tratamiento debidamente justificada y más adecuada al contexto local, debería permitir tanto una operación menos costosa como un saneamiento en aguas residuales más sostenible y universal en Costa Rica. Ambas propuestas fueron establecidas en la Política Nacional de Saneamiento (AyA et al., 2016) que se espera guíe el desarrollo del saneamiento en aguas residuales costarricenses para los próximos 25 años.

Esfuerzos adicionales en la investigación y experimentación con tecnologías menos conocidas en el país, como los sistemas anaerobios con un postratamiento aerobio, podrían representar una oportunidad para la promoción de un saneamiento universal y sostenible en Costa Rica, al igual que otras regiones tropicales de América Latina.

\section{Agradecimientos}

Se agradece a la arquitecta Kathy Borges Umaña y a la ingeniera Natalia Torres Corral, por facilitar la información de la base de datos dirigida a los proyectos tramitados en el APC, para su aprobación por parte del AyA. También, a la Revista y las personas revisoras anónimas, por sus oportunos comentarios para mejorar la versión final de este escrito.

\section{Referencias}

Angulo, F. (2014). Gestión del recurso hídrico y saneamiento en Costa Rica. En Programa del Estado de la Nación, Vigésimo primer Informe del Estado de la Nación en Desarrollo Humano Sostenible (p. 47). Costa Rica: PEN.

\begin{tabular}{|c|c|c|}
\hline 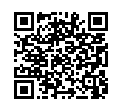 & (c) & 108 \\
\hline
\end{tabular}




\section{Revista de CIENCIAS AMBIENTALES Tropical Journal of Environmental Sciences}

Revista de Ciencias Ambientales (Trop J Environ Sci) e-ISSN: 2215-3896

(Julio-Diciembre, 2019) . Vol 53(2): 97-110 DOI: https://doi.org/10.15359/rca.53-2.5 Open Access: www.revistas.una.ac.cr/ambientales e-mail: revista.ambientales@una.ac.cr Centeno E. y Murillo A.

Arias, A. L. (2010). Sector Agua Potable y Saneamiento. En Programa del Estado de la Nación, Decimoséptimo Informe del Estado de la Nación (p. 11). Costa Rica: PEN.

Astorga, Y. y Angulo, F. (2013). Gestión del recurso hídrico y saneamiento. En Programa del Estado de la Nación, Vigésimo Informe Estado de la Nación en Desarrollo Humano Sostenible (p. 29). Costa Rica: PEN.

AyA, MINAE y MINSA. (2016). Política Nacional de Saneamiento en Aguas Residuales. Costa Rica: CR.

Campos, A. (2015). Experiencias institucionales en el uso del Sistema APC para la aprobación de plantas de tratamiento de aguas residuales Resumen Introducción. Hidrogénesis, 11(1), 97-109.

Chernicharo, C. A. L. (2016). Reatores anaeróbios. Brasil: UFMG

Chernicharo, C. A. L., van Lier, J. B., Noyola, A. y Bressani Ribeiro, T. (2015). Anaerobic sewage treatment: state of the art, constraints and challenges. Reviews in Environmental Science and Biotechnology, 14(4), 649-679. https://doi.org/10.1007/s11157-015-9377-3

DE-33601-S-Minae. (2007). Reglamento de vertido y reuso de aguas residuales. Costa Rica: CR.

Herrera, J. (2016). Recurso hídrico y saneamiento: avances y desafíos. En Programa del Estado de la Nación, Vigésimo segundo Informe del Estado de la Nación en Desarrollo Humano Sostenible (vol. 1, p. 34). Costa Rica: PEN.

Herrera, J. (2017). Uso y estado de los recursos: recurso hídrico. En Programa del Estado de la Nación, Informe del Estado de la Nación en Desarrollo Humano Sostenible (p. 32). Costa Rica: PEN.

Lema, J. y Suárez-Martínez, S. (2017). Innovative Wastewater Treatment \& Resource Recovery Technologies. Reino Unido: IWA Publishing.

Metcalf y Eddy. (2014). Wastewater engineering: treatment and resource recovery (5. $\left.{ }^{\mathrm{a}} \mathrm{ed}.\right)$. Estados Unidos: McGrawHill.

Mora, D., Mata, A. y Portuguez, C. (2016). Agua para consumo humano y saneamiento y su relación con los indicadores básicos de salud en Costa Rica: Objetivos de desarrollo del milenio y la agenda para el 2030. Costa Rica: AyA.

Murillo, A. (2017). Análisis comparativo de tecnologías para el tratamiento colectivo de las aguas residuales en poblaciones menores a 5000 habitantes equivalentes en Costa Rica (Tesis de pregrado). Universidad de Costa Rica, San José, Costa Rica.

\begin{tabular}{|c|c|c|}
\hline 品被 & (c) (i) (-) & 109 \\
\hline
\end{tabular}




\section{Revista de CIENCIAS AMBIENTALES Tropical Journal of Environmental Sciences}

Revista de Ciencias Ambientales (Trop J Environ Sci) e-ISSN: 2215-3896

(Julio-Diciembre, 2019) . Vol 53(2): 97-110 DOI: https://doi.org/10.15359/rca.53-2.5 Open Access: www.revistas.una.ac.cr/ambientales e-mail: revista.ambientales@una.ac.cr Centeno E. y Murillo A.

Noyola, A., Padilla-Rivera, A., Morgan-Sagastume, J. M., Güereca, L. P. y Hernández-Padilla, F. (2012). Typology of Municipal Wastewater Treatment Technologies in Latin America. Clean - Soil, Air, Water, 40(9), 926-932. https://doi.org/10.1002/clen.201100707

Rodríguez-García, G., Molinos-Senante, M., Hospido, A., Hernández-Sancho, F., Moreira, M. T. y Feijoo, G. (2011). Environmental and economic profile of six typologies of wastewater treatment plants. Water Research, 45(18), 5997-6010. https://doi.org/10.1016/j.watres.2011.08.053

UN WATER. (2017). Informe Mundial de las Naciones Unidas sobre el Desarrollo de los Recursos Hídricos 2017. Aguas residuales, el recurso desaprovechado. Francia: UNESCO.

Sperling, M. y Chernicharo, C. A. L. (2005). Biological Wastewater Treatment in Warm Climate Regions. Brasil: IWA Publishing.

Sperling, M. (2014). Introdução à qualidade das águas e ao tratamento de esgotos (4. ${ }^{\mathrm{a}}$ ed.). Brasil: UFMG.

Sperling, M. y Chernicharo, C. A. L. (2002). Urban wastewater treatment technologies and the implementation of discharge standards in developing countries. Urban Water, 4(1), 105-114. https://doi.org/10.1016/S1462-0758(01)00066-8

WEF y ASCE. (2017). Design of Water Resource Recovery Facilities (6. ${ }^{\mathrm{a}}$ ed.). Estados Unidos: McGraw-Hill Education.

\begin{tabular}{|c|c|c|c|c|c|}
\hline 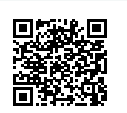 & (c) (i) (-) & $\underset{\text { AMBENTAIES }}{\longrightarrow}$ & $\frac{10 \%}{20}$ & 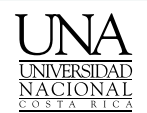 & 110 \\
\hline
\end{tabular}

